\title{
Decoherent Histories and the Emergent Classicality of Local Densities
}

\author{
J.J.HALLIWELL \\ Theory Group \\ Blackett Laboratory \\ Imperial College \\ London \\ SW7 2BZ \\ $U K$
}

Imperial Preprint/TP/98-99/53. Revised version. August, 1999

\begin{abstract}
In the context of the decoherent histories approach to quantum theory, it is shown that a class of macroscopic configurations consisting of histories of local densities (number, momentum, energy) exhibit negligible interference. This follows from the close connection of the local densities with the corresponding exactly conserved (and so exactly decoherent) quantities, and also from the observation that the eigenstates of local densities (averaged over a sufficiently large volume) remain approximate eigenstates under time evolution. The result is relevant to the derivation of hydrodynamic equations using the decoherent histories approach.
\end{abstract}


The key step in many studies of emergent classicality from quantum mechanics is the demonstration that certain types of quantum states of the system exhibit negligible interference. Initial superpositions of such states may therefore be effectively replaced by statistical mixtures. This, loosely speaking, is decoherence, and has principally been demonstrated for the situation in which there is a distinguished system, such as a particle, coupled to its surrounding environment [1].

Most generally, decoherence typically comes about when the variables describing the entire system of interest naturally separate into "slow" and "fast", whether or not this separation corresponds to, respectively, system and environment ${ }^{\dagger}$. If the system consists of a large collection of interacting identical particles, as in a fluid for example, the natural set of slow variables are the local densities: energy, momentum, number, charge etc. These variables, in fact, are also the variables which provide the most complete description of the classical state of a fluid at a macroscopic level. The most general demonstration of emergent classicality therefore consists of showing that, for a large collection of interacting particles described microscopially by quantum theory, the local densities become effectively classical. Although one might argue that the system-environment mechanism might play a role, since the collection of particles are coupled to each other, decoherence comes about in these situations for a different reason: it is because the local densities are almost conserved if averaged over a sufficiently large volume [3]. Hence, the approximate non-interference of local densities is due to the fact that they are close to a set of exactly conserved quantities, and exactly conserved quantities obey superselection rules.

Intuitively appealing though this argument is, it is clearly a quantitative issue. The object of this letter is to show that, under certain reasonable conditions, local densities

$\dagger$ See Ref.[2] for a discussion of the conditions under which the total Hilbert space may be written as a tensor product of system and environment Hilbert spaces 
averaged over a sufficiently large volume are indeed approximately decoherent as a result of their close connection to exact conservation.

We will approach the question using the decoherent histories approach to quantum theory [3-5], which has proved particularly useful for discussing emergent classicality in a variety of contexts*. The central object of interest is the decoherence functional,

$$
D\left(\underline{\alpha}, \underline{\alpha}^{\prime}\right)=\operatorname{Tr}\left(C_{\underline{\alpha}}|\Psi\rangle\left\langle|\Psi| C_{\underline{\alpha}^{\prime}}^{\dagger}\right)\right.
$$

The histories are characterized by the initial state $|\Psi\rangle$ and by the time-ordered strings of projection operators $C_{\underline{\alpha}}=P_{\alpha_{n}}\left(t_{n}\right) \cdots P_{\alpha_{1}}\left(t_{1}\right)$ (where $\underline{\alpha}$ denotes the string of alternatives $\left.\alpha_{1}, \alpha_{2} \cdots \alpha_{n}\right)$. Intuitively, the decoherence functional is a measure of the interference between pairs of histories $\underline{\alpha}, \underline{\alpha}^{\prime}$. When it is zero for $\underline{\alpha} \neq \underline{\alpha}^{\prime}$, we say that the histories are decoherent and probabilities $p(\underline{\alpha})=D(\underline{\alpha}, \underline{\alpha})$ obeying the usual probability sum rules may be assigned to them. Although not addressed here, one can then ask whether these probabilities are strongly peaked about trajectories obeying classical equations of motion. For the local densities, these equations will be hydrodynamic equations, and these and closely related aspects of emergent classicality have been pursued at greater length elsewhere Refs. $[2,7-9]$.

We consider the class of systems which are described at the microscopic level by a Hamiltonian of the form

$$
H=\sum_{j}\left(\frac{\mathbf{p}_{j}^{2}}{2 m}+\sum_{\ell>j} \phi\left(\left|\mathbf{q}_{j}-\mathbf{q}_{\ell}\right|\right)\right)
$$

For definiteness, we will concentrate on the case of a dilute gas with short-range interactions, but it will be clear that the physical ideas are reasonably general. The local

* The extent to which the approach fully explains emergent classicality has been criticized [6]. This paper concerns the mathematical properties of the approach, as it currently stands, and adds nothing to that debate. See Ref.[7] and references therein for further discussion. 
densities of interest are the number density $n(\mathbf{x})$, the momentum density $\mathbf{g}(\mathbf{x})$ and the energy density $h(\mathbf{x})$, defined by,

$$
\begin{aligned}
& n(\mathbf{x})=\sum_{j} \delta\left(\mathbf{x}-\mathbf{q}_{j}\right) \\
& \mathbf{g}(\mathbf{x})=\sum_{j} \mathbf{p}_{j} \delta\left(\mathbf{x}-\mathbf{q}_{j}\right) \\
& h(\mathbf{x})=\sum_{j}\left(\frac{\mathbf{p}_{j}^{2}}{2 m}+\sum_{\ell>j} \phi\left(\left|\mathbf{q}_{j}-\mathbf{q}_{\ell}\right|\right)\right) \delta\left(\mathbf{x}-\mathbf{q}_{j}\right)
\end{aligned}
$$

(suitably ordered, in the quantum case). We are interested in local densities smeared over a volume $V$. The effect of this is to replace the delta functions with a window function, denoted $\delta_{V}$, which is zero outside $V$ and 1 inside. It is also useful to work with the Fourier transforms of the local densities, denoted $n(\mathbf{k}), g(\mathbf{k}), h(\mathbf{k})$. So, for example, the local number density at wavelength $\mathbf{k}$ is

$$
n(\mathbf{k})=\sum_{j} e^{i \mathbf{k} \cdot \mathbf{q}_{j}}
$$

Exact conservation is obtained in the limit $k=|\mathbf{k}| \rightarrow 0$, or $V \rightarrow \infty$ in (3)-(5).

We would like to compute the decoherence functional for histories consisting of projections onto the operators $(3)-(5)$. (The construction of the projectors is described in more detail in Ref.[7]). In the case of exact conservation, $k=0$, we have exact decoherence simply because the projectors in Eq.(1) all commute with $H$ and with each other [10]. Our main task is therefore to show that as $k$ increases from zero there is still a non-trivial regime in which decoherence is approximately maintained. A significant result of this type has been established already by Calzetta and $\mathrm{Hu}$ for the case of local temperature $T(x)$ obeying the diffusion equation [9]. They took their initial state to be close to the equilibrium state, whereas here, by contrast, initial macroscopic superposition states are considered. 
We begin by rewriting the exact conservation case in a simple way that makes its generalization to locally conserved quantities more apparent. Suppose the histories are projections onto some conserved quantity, $Q$. Let the initial state be a superposition of eigenstates of $Q$,

$$
|\Psi\rangle=\frac{1}{\sqrt{2}}(|a\rangle+|b\rangle)
$$

where $\langle a \mid b\rangle=0$ and

$$
\hat{Q}|a\rangle=a|a\rangle, \quad \hat{Q}|b\rangle=b|b\rangle
$$

Since the $P_{\alpha}$ 's are projections onto $Q, P_{\alpha}$ either annihilates or preserves $|a\rangle$ and $|b\rangle$. Take the case of a history with just two moments of time (the generalization to more times is trivial). The only non-zero off-diagonal terms of the decoherence functional are of the form

$$
\begin{aligned}
D\left(\underline{\alpha}, \underline{\alpha}^{\prime}\right) & =\frac{1}{2} \operatorname{Tr}\left(P_{\alpha_{2}} e^{-\frac{i}{\hbar} H t}|a\rangle\langle b| e^{\frac{i}{\hbar} H t}\right) \\
& =\frac{1}{2} \operatorname{Tr}\left(P_{\alpha_{2}}\left|a_{t}\right\rangle\left\langle b_{t}\right|\right)
\end{aligned}
$$

But $Q$ is conserved, hence $\left[P_{\alpha_{2}}, H\right]=0$ and

$$
\begin{aligned}
P_{\alpha_{2}}\left|a_{t}\right\rangle & =P_{\alpha_{2}} e^{-\frac{i}{\hbar} H t}|a\rangle \\
& =e^{-\frac{i}{\hbar} H t} P_{\alpha_{2}}|a\rangle=\left|a_{t}\right\rangle
\end{aligned}
$$

(or equals zero if $\alpha_{2}$ does not correspond to $a$ ). It follows that

$$
\begin{aligned}
D\left(\underline{\alpha}, \underline{\alpha}^{\prime}\right) & =\frac{1}{2} \operatorname{Tr}\left(P_{\alpha_{2}}\left|a_{t}\right\rangle\left\langle b_{t}\right|\right) \\
& =\left\langle b_{t} \mid a_{t}\right\rangle=\langle b \mid a\rangle=0
\end{aligned}
$$

and therefore we have decoherence.

Now suppose that the operator $Q$ is one of the local densities $(3)-(5)$, so is no longer exactly conserved. The steps up to Eq.(9) still hold. But to go further, we need to 
know how the eigenstates of the local densities behave under time evolution. A reasonable supposition, which will be justified, is the following. Let us suppose that under time evolution, the eigenstates of $Q$ remain approximate eigenstates. That is, we initially have (7), but under evolution to time $t, \hat{Q}\left|a_{t}\right\rangle \approx\langle Q\rangle\left|a_{t}\right\rangle$ or, more precisely,

$$
\frac{(\Delta Q)^{2}}{\langle Q\rangle^{2}}<<1
$$

i.e., the state remains strongly peaked in the variable $Q$ under time evolution. The states are then approximate eigenstates of the projectors, so that in place of Eq.(10), we have the approximate result, $P_{\alpha_{2}}\left|a_{t}\right\rangle \approx\left|a_{t}\right\rangle$ (or equals zero) as long as the width of the projection is much greater than the uncertainty $(\Delta Q)^{2}$. Hence Eq.(11) follows approximately, and we get approximate decoherence to the extent that the approximation (12) holds.

The key point is therefore the following: approximate decoherence is assured for histories of operators $Q$ whose eigenstates have the property that they remain strongly peaked in $Q$ under time evolution, as characterized by (12). To demonstrate decoherence of the local densities, therefore, we need only find their eigenstates, and show that they satisfy the localization property (12) under time evolution. (Note, incidently, that the above argument actually assures decoherence of any variables $Q$ satisfying the localization property. The particular significance of the local densities is that they are continuous functions of the coarse graining scale $k$, so are guaranteed to satisfy the requisite property if $k$ is sufficiently close to zero.)

Since the three operators (3)-(5) do not commute, exact simultaneous eigenstates cannot be found. However, a useful class of approximate eigenstates of all three operators are the states consisting of products of $N$ identical terms,

$$
|\Psi\rangle=|\psi\rangle \otimes|\psi\rangle \otimes \cdots \otimes|\psi\rangle
$$


These may be shown to be eigenstates (of the local number density, for example) by observing that the object $(\Delta n(\mathbf{x}))^{2} /\langle n(\mathbf{x})\rangle^{2}$ goes like $1 / N$ for large $N$ (see Ref.[7], for example). It is essentially the central limit theorem (see also Ref.[11]). For the number and momentum density it relies on the fact that they are sums of identical one-particle operators. For the local energy density, it additionally requires the smearing volume to be sufficiently large, compared to some lengthscale indicated by the interactions. Some tuning of the state $|\psi\rangle$ can be carried out to ensure that (13) is an optimal approximate eigenstate of all the local densities but this will not be done here. (Also, the passage to exact eigenstates of $n(\mathbf{k}), g(\mathbf{k}), h(\mathbf{k})$ as $k \rightarrow 0$ can be seen explicitly if the one-particle states $|\psi\rangle$ are taken to be one-particle momentum eigenstates).

The question is now what happens to the eigenstates (13) of the local densities under time evolution by the Hamiltonian (2). Consider first the trivial but enlightening case in which there no interactions. In this case, the time evolved eigenstates $\left|a_{t}\right\rangle$ remain of the product form (13), so they are still approximate eigenstates of the local densities (but with a time-evolved eigenvalue) for the same reasons as above. Hence there is approximate decoherence.

Decoherence in the non-interacting case comes about for two reasons. First, it is due to the fact that a state of the form (13) will remain strongly peaked about the average values of the local densities, $n(\mathbf{x}), g(\mathbf{x}), h(\mathbf{x})$ under time evolution, and thus the state is essentially undisturbed by the projectors (as long as their widths are sufficiently large). Secondly, it is due to the almost trivial fact that the orthogonality of the two elements of the initial state is preserved by unitary evolution. This second fact is important because the first one is not always sufficient to guarantee decoherence. Although the state remains strongly peaked about the average values of the local densities, these average values do not necessarily obey deterministic equations. In the case of histories characterized by number 
density only, for example, $\langle n(\mathbf{x})\rangle$ at time $t$ is not uniquely determined by $\langle n(\mathbf{x})\rangle$ at the initial time (in the state (13)). That is, in Eq.(9), $\left|a_{t}\right\rangle$ and $\left|b_{t}\right\rangle$ may in fact be peaked about the same value of number density, even though the initial values are different. The decoherence is therefore not in fact due to an approximate determinism (such as that used in the phase space histories of Omnès [5]). It is necessary only that the evolved states are essentially undisturbed by the projectors, and therefore that the two orthogonal components of the initial state are eventually overlapped at the final time, as in Eq.(11), to give zero.

The next and most important task is to show that the above story is in fact still true, with qualifications, in the presence of interactions. The complete description of $N$ interacting particles is complicated but we can make some progress by making two assumptions which are standard in kinetic theory [12]. It is notationally convenient in what follows to work with a Wigner function, rather than quantum state. Hence associated with the full $N$-particle wave function is an $N$-particle Wigner function $W_{N}\left(\mathbf{p}_{1}, \mathbf{q}_{1}, \cdots \mathbf{p}_{N}, \mathbf{q}_{N}\right)$. Our first assumption is that the three-particle correlations are negligible. It means that all the physics is contained in the one and two-particle reduced Wigner functions, $W_{1}\left(\mathbf{p}_{1}, \mathbf{q}_{1}\right)$ and $W_{2}\left(\mathbf{p}_{1}, \mathbf{q}_{1}, \mathbf{p}_{2}, \mathbf{q}_{2}\right)$.

We again take as our initial state the approximate eigenstate (13), and let it evolve, so correlations will develop. The degree to which the particles become correlated is contained in the two-particle distribution $W_{2}$ of the evolved eigenstate. On general grounds, we expect that the inter-particle correlations will only be important on some length scale $L$, and beyond that length scale, they will be uncorrelated. That is, we will assume that

$$
W_{2}\left(\mathbf{p}_{1}, \mathbf{q}_{1}, \mathbf{p}_{2}, \mathbf{q}_{2}\right) \approx W_{1}\left(\mathbf{p}_{1}, \mathbf{q}_{1}\right) W_{1}\left(\mathbf{p}_{2}, \mathbf{q}_{2}\right)
$$

for $\left|\mathbf{q}_{2}-\mathbf{q}_{1}\right|>L$, and otherwise $W_{2}$ will have a form indicating non-trivial correlations. 
This is our second assumption. It is physically reasonable for uncorrelated initial states of the form (13) with a short range interaction (and it is in fact a key assumption in the derivation of the Boltzmann equation [12]). It would not of course be an appropriate assumption for correlated initial states, but the point is that we are interested in approximate eigenstates of the local densities, and a useful class of such states have the uncorrelated from (13).

Given the above assumptions, it is now reasonably straighforward to argue that the state is still strongly peaked about the average values of the local densities, as long as $V>>L^{3}$. For example, for the number density, we have

$$
\langle n(\mathbf{x})\rangle=\sum_{j}\left\langle\delta_{V}\left(\mathbf{q}_{j}-\mathbf{x}\right)\right\rangle=N \int_{V} d^{3} \mathbf{q} p(\mathbf{q})
$$

where $p(\mathbf{q})$ is the one-particle probability distribution of $\mathbf{q}$ (obtained by integrating the one-particle Wigner function over $\mathbf{p})$. Similarly,

$$
\begin{aligned}
\left\langle n^{2}(\mathbf{x})\right\rangle & =\sum_{j \ell}\left\langle\delta_{V}\left(\mathbf{q}_{j}-\mathbf{x}\right) \delta_{V}\left(\mathbf{q}_{\ell}-\mathbf{x}\right)\right\rangle \\
& =N\left\langle\delta_{V}\right\rangle+\left(N^{2}-N\right)\left\langle\delta_{V}\left(\mathbf{q}_{1}-x\right) \delta_{V}\left(\mathbf{q}_{2}-x\right)\right\rangle
\end{aligned}
$$

where we have used $\delta_{V}^{2}=\delta_{V}$, and also an assumption of identical particles to reduce the sum over $j, \ell$ to particles labeled 1 and 2 . We now have

$$
\begin{aligned}
(\Delta n(\mathbf{x}))^{2}= & \left\langle n^{2}(\mathbf{x})\right\rangle-\langle n(\mathbf{x})\rangle^{2} \\
= & N^{2}\left(\left\langle\delta_{V}\left(\mathbf{q}_{1}-\mathbf{x}\right) \delta_{V}\left(\mathbf{q}_{2}-\mathbf{x}\right)\right\rangle-\left\langle\delta_{V}\right\rangle^{2}\right) \\
& +N\left(\left\langle\delta_{V}\right\rangle-\left\langle\delta_{V}\left(\mathbf{q}_{1}-\mathbf{x}\right) \delta_{V}\left(\mathbf{q}_{2}-\mathbf{x}\right)\right\rangle\right)
\end{aligned}
$$

If there is no correlation at all between the particles, the coefficient of $N^{2}$ would vanish, so $(\Delta n(\mathbf{x}))^{2} /\langle n(\mathbf{x})\rangle^{2}$ would go like $1 / N$, which goes to zero as $N \rightarrow \infty$. This is the standard central limit theorem result indicated earlier for the non-interacting case. With 
interactions, the coefficient of $N^{2}$ is no longer zero. We now need to show, therefore, that this term is still sufficiently small for $(\Delta n(\mathbf{x}))^{2} /\langle n(\mathbf{x})\rangle^{2}$ to remain small as $N \rightarrow \infty$. Introducing the two-particle distribution $p\left(\mathbf{q}_{1}, \mathbf{q}_{2}\right)$ (obtained by integrating $\mathbf{p}_{1}, \mathbf{p}_{2}$ out of $W_{2}$ ), it is readily shown that the leftover terms as $N \rightarrow \infty$ are

$$
\frac{(\Delta n(\mathbf{x}))^{2}}{\langle n(\mathbf{x})\rangle^{2}}=\frac{\int_{V} d^{3} \mathbf{q}_{1} \int_{V} d^{3} \mathbf{q}_{2}\left(p\left(\mathbf{q}_{1}, \mathbf{q}_{2}\right)-p\left(\mathbf{q}_{1}\right) p\left(\mathbf{q}_{2}\right)\right)}{\left(\int_{V} d^{3} \mathbf{q} p(\mathbf{q})\right)^{2}}
$$

This is clearly zero if there are no correlations. In the interacting case we use the assumption (14), which implies that

$$
p\left(\mathbf{q}_{1}, \mathbf{q}_{2}\right) \approx p\left(\mathbf{q}_{1}\right) p\left(\mathbf{q}_{2}\right)
$$

for $\left|\mathbf{q}_{1}-\mathbf{q}_{2}\right|>L$, and otherwise non-trivial correlations exist. Hence the integral in the numerator takes contributions only from the region $\left|\mathbf{q}_{1}-\mathbf{q}_{2}\right|<L$.

To see that (18) is small, note that in the numerator, the integral is over a volume $V^{2}$ in the six-dimensional two particle configuration space. If $V<<L^{3}$, the factorization of $p\left(\mathbf{q}_{1}, \mathbf{q}_{2}\right)$ for $\left|\mathbf{q}_{1}-\mathbf{q}_{2}\right|>L$ makes no difference, since $\mathbf{q}_{1}$ and $\mathbf{q}_{2}$ can never be far enough apart in the integrand (assuming $V$ is regular in shape). However, if $V>>L^{3}$, the $V^{2}$ sized integration region is substantially reduced in size to $V \times L^{3}$. On dimensional grounds the numerator is therefore proportional to a number of order $V L^{3}$, and the denominator to $V^{2}$ (perhaps with other factors common to both). This means that

$$
\frac{(\Delta n(\mathbf{x}))^{2}}{\langle n(\mathbf{x})\rangle^{2}} \sim \frac{L^{3}}{V}
$$

This order of magnitude estimate becomes exact if we assume that the probabilities are constant in the region of non-trivial correlation (another common assumption of kinetic theory [12]). Hence the state will be strongly peaked about the average of $n(\mathbf{x})$ if $V>>L^{3}$.

It is possible to see on physical grounds why one expects a result of the form (20) to hold quite generally. In the non-interacting case we used the central limit theorem result that 
$(\Delta n)^{2} /\langle n\rangle^{2}$ goes like $1 / N$. In the interacting case, the state is no longer of the product form (13), but an analagous result still holds. The point is that the correlations that develop extend only over a (typically small) volume of size $L^{3}$, so the system breaks up into a large number of essentially identical uncorrelated regions of this size. Therefore each smearing volume $V$, if much greater than $L^{3}$, contains of order $V / L^{3}$ identical uncorrelated regions each of which contribute equally to the local density averaged over $V$. Loosely speaking, a central limit theorem-type result again applies, not to the $N$ uncorrelated particles in the same state, but to the $V / L^{3}$ uncorrelated regions. So $1 / N$ is replaced by $L^{3} / V$ in the central limit theorem, and hence the above result.

Similar results hold for the local momentum and energy density. We have therefore demonstrated the desired result: a class of eigenstates of the coarse-grained local densities remain approximate eigenstates under time evolution as long as the smearing volume is much greater than the correlation volume of these states. Decoherence of these variables then follows. More details of this work, including a discussion of the approach to local equilibrium and the emergence of hydrodynamic evolution equations, will be published elsewhere [13].

Acknowledgements: I am grateful to Todd Brun, Jim Hartle, Ray Rivers and Tom Kibble for useful conversations.

\section{References}

1. E.Joos and H.D.Zeh, Z.Phys. B59, 223 (1985); W.Zurek, in Physical Origins of Time Asymmetry, edited by J.J.Halliwell, J.Perez-Mercader and W.Zurek (Cambridge University Press, Cambridge, 1994); preprint quant-ph/9805065.

2. T.Brun and J.B.Hartle, quant-ph/9905079. 
3. M.Gell-Mann and J.B.Hartle, in Complexity, Entropy and the Physics of Information, SFI Studies in the Sciences of Complexity, Vol. VIII, W. Zurek (ed.) (Addison Wesley, Reading, 1990); Phys.Rev. D47, 3345 (1993).

4. R.B.Griffiths, J.Stat.Phys. 36, 219 (1984); Phys.Rev.Lett. 70, 2201 (1993).

5. R. Omnès, J.Stat.Phys. 53, 893 (1988); 53, 933 (1988); 53, 957 (1988); 57, 357 (1989); Ann.Phys. 201, 354 (1990); Rev.Mod.Phys. 64, 339 (1992).

6. H.F.Dowker and A.Kent, J.Stat.Phys. 82, 1575 (1996); Phys.Rev.Lett. 75, 3038 (1995); A. Kent, Phys.Rev. A54, 4670 (1996).

7. J.J.Halliwell, Phys.Rev. D58, 105015 (1998).

8. C.Anastopoulos, preprint gr-qc/9805074 (1998); T.Brun and J.J.Halliwell, Phys.Rev. 54, 2899 (1996); E. Calzetta and B. L. Hu, in Directions in General Relativity, edited by B. L. Hu and T. A. Jacobson (Cambridge University Press, Cambridge, 1993).

9. E.A.Calzetta and B.L.Hu, Phys.Rev. D59, 065018 (1999).

10. J. B. Hartle, R. Laflamme and D. Marolf, Phys.Rev.D51, 7007 (1995).

11. J.B.Hartle, Am.J.Phys. 36, 704 (1968).

12. See for example, R.L.Liboff, Introduction to the Theory of Kinetic Equations (Wiley, New York, 1969); K.Huang, Statistical Mechanics, 2nd edition (New York, Chichester, Wiley, 1987).

13. J.J.Halliwell and J.B.Hartle (in preparation). 